\title{
Does contrast-enhanced mammography have an impact on the detection of cancer in patients with risk of developing breast cancer?
}

\author{
Lamiaa Mohamed Bassam Hashem ${ }^{1,2^{*}}$ (D), Nancy Osama Abd El Hamid ${ }^{3}$, Rasha Mohamed Kamal 1,2, \\ Sahar Mahmoud Mansour ${ }^{1,2}$, Shaimaa Lasheen ${ }^{4}$ and Yasmin Mounir Tohamey ${ }^{1,2}$
}

\begin{abstract}
Background: Contrast-enhanced mammography (CEM) has been discovered to be more sensitive and specific than two-dimensional full-field digital mammography (FFDM) in both screening and diagnostic settings. The aim of the study was to assess the additive role of CEM in the detection and characterization of breast lesions in women with increased risk of developing breast cancer. This prospective study included 283 female patients with increased risk of developing breast cancer (i.e., positive family history of breast cancer, personal history of breast cancer, and heterogeneously dense mammary parenchyma) coming for either screening $(n=127 / 283(49.1 \%))$ or diagnostic $(n=156 / 283$ (55.1\%)) purpose. All patients had FFDM and CEM done, and the findings were evaluated independently; final Breast Imaging Reporting And Data System (BIRADS) classification was given for each modality. Results were then compared with histopathology or ultrasound findings with routine follow-up for normal and typically benign findings.

Results: In this study, 283 women with mean age of 48 were enrolled. Among the studied cases regardless to a specific risk factor, 15/283 (5.3\%) were diagnosed as normal, 13/283 (4.6\%) as inflammatory lesions, 72/283(25.4\%) as benign lesions, 6/283 (2.1\%) as benign precancerous lesions, and 177/283 (62.5\%) as malignant. The overall sensitivity and specificity of the CEM were 92.7 and $71.43 \%$, respectively, while FFDM were 80.90 and 59.05\%, respectively.

Conclusion: Contrast-enhanced mammography is a valuable screening and diagnostic imaging modality in patients with increased risk of developing breast cancer with diagnostic indices higher than mammography resulting in a significantly higher cancer detection rate.
\end{abstract}

Keywords: Mammography, Risk factors, Breast cancer, Higher-risk population, Early detection of cancer

\footnotetext{
* Correspondence: lamia.bassam22@gmail.com

'Department of Diagnostic and Interventional Radiology, Faculty of

Medicine, Cairo University, Cairo, Egypt

${ }^{2}$ Department of Diagnostic and Interventional Radiology, Baheya Foundation,

Cairo, Egypt

Full list of author information is available at the end of the article
}

\section{Springer Open}

(c) The Author(s). 2021 Open Access This article is licensed under a Creative Commons Attribution 4.0 International License, which permits use, sharing, adaptation, distribution and reproduction in any medium or format, as long as you give appropriate credit to the original author(s) and the source, provide a link to the Creative Commons licence, and indicate if changes were made. The images or other third party material in this article are included in the article's Creative Commons licence, unless indicated otherwise in a credit line to the material. If material is not included in the article's Creative Commons licence and your intended use is not permitted by statutory regulation or exceeds the permitted use, you will need to obtain permission directly from the copyright holder. To view a copy of this licence, visit http://creativecommons.org/licenses/by/4.0/. 


\section{Background}

In the last decades, screening mammography programs have led to increase in the detection and early diagnosis of breast cancer [1]. Cancer grows when deoxyribonucleic acid (DNA) is damaged, but the exact reason it becomes damaged is still unknown. However, there are certain factors that are linked to and may increase the incidence of breast cancer [2].

Every woman is at some risk that ranges from low to high for breast cancer. Women with increased breast density are doubly unlucky; they are at higher risk of developing breast cancer and at greater risk that cancer will be undetectable and radiologically masked by increased density [3].

Even though, mammography plays an important role in screening and diagnosis of breast cancer, imaging approach has changed over time to a more personalized, risk-based approach [4]. The current breast cancer screening recommendations state that for patients at high risk of developing breast cancer, an annual screening magnetic resonance imaging (MRI) is appropriate in addition to an annual mammogram [5].

Contrast-enhanced mammography (CEM) is an advanced technology arising from the digital mammography platform. The theory behind it is based on the success of the most sensitive of all breast imaging techniques; contrast-enhanced MRI (CE-MRI) [6]. Results of clinical studies conclude that CEM has sensitivity and specificity comparable with breast CE-MRI [7].

So the aim of this work was to assess the additive role of CEM in the detection and characterization of breast lesions in women with risk of developing breast cancer.

\section{Methods}

\section{Patients}

This study included 283 cases with increased risk of developing breast cancer either presented for diagnostic breast imaging $(n=156 / 283(55.1 \%))$ or referred from the screening breast unit $(n=127 / 283(49.1 \%))$ during the period between October 2017 and July 2018. Their ages ranged from 19-75 years (mean age $48.5 \pm 11.5$ ).

\section{Inclusion criteria}

All patients had moderate or high risk of developing breast cancer based on the ACR criteria [5]. They had at least one of the following: (1) single sonomammographic suspicious lesion; BIRADS 4 and 5 (Mass with indistinct margins and irregular shape on sonomammography, micro-calcifications of suspicious morphology, architecture distortion, focal asymmetry, and complex breast cysts), (2) axillary lymphadenopathy with no detectable lesions on mammogram or breast ultrasound, or (3) dense breasts.

\section{Exclusion criteria}

We excluded patients with average risk of developing breast cancer (general normal population who do not have moderate or high risk of developing breast cancer) and those who had contraindication to mammography and IV contrast.

\section{All patients were subjected to demographic and clinical data collection}

Full history taking was done including patient's name, age, marital status, and number of offsprings, lactation history, residence and phone number, diagnosis, duration of illness, past medical history, and family history. Data are shown in Table 1.

\section{Diagnostic tools}

All patients $(n=283 / 283)$ had a reported sonomammography and underwent CEM. The diagnostic indication for CEM in our study was better lesion detection and/or lesion characterization, thus consequently accurate BIRADS classification.

\section{Technique of CEM}

Contrast-enhanced mammography examination was performed using Senographe Essential, GE healthcare FFDM machine. First, a one-shot intravenous injection of a non-ionic contrast media $(1.5 \mathrm{~mL} / \mathrm{Kg})$ (injection rate of $3 \mathrm{~mL} / \mathrm{s}$ ) was performed through a cannula inserted in the antecubital vein (contralateral to the side of concern, if any). Two minutes after contrast administration, each breast was compressed in both the cranio-caudal (CC) position and the mediolateral oblique (MLO) view, and a pair (low and high energy) of images were acquired within $20 \mathrm{~s}$ of one another. By subtraction of the $2 \mathrm{im}$ ages, through appropriate image processing, two iodineenhanced images were generated-one in MLO and the other in $\mathrm{CC}$ projection.

Table 1 Demographic and clinical data of the studied cases

\begin{tabular}{lll}
\hline Factors & Yes & No \\
\hline Age 40 and above & $222 / 283(78.4 \%)$ & $61 / 283(21.6 \%)$ \\
Breast density & $259 / 283(91.5 \%)$ & $24 / 283(8.5 \%)$ \\
Family history & $84 / 283(29.7 \%)$ & $199 / 283(70.3 \%)$ \\
Married & $261 / 283(92.2 \%)$ & $22 / 283(7.8 \%)$ \\
Children & $232 / 283(82 \%)$ & $51 / 283(18 \%)$ \\
Breastfeeding & $239 / 283(84.5 \%)$ & $44 / 283(15.5 \%)$ \\
Contraception & $73 / 283(25.8 \%)$ & $210 / 283(74.2 \%)$ \\
Menopause & $107 / 283(37.8 \%)$ & $176 / 283(62.2 \%)$ \\
Previous operation & $16 / 283(5.7 \%)$ & $267 / 283(94.3 \%)$ \\
(malignant or precancerous) & & \\
Complaint & $156 / 283(55.1 \%)$ & $127 / 283(49.9 \%)$ \\
\hline
\end{tabular}




\section{Image analysis}

Image analysis and interpretation were done by a single consultant radiologist with more than 10 years of experience in breast imaging field, guided by the results of clinical data but blind to final pathologic diagnosis or previous sonomammography results.

\section{Image analysis and interpretation of CEM}

Following Kamal et al. [8], contrast-enhanced mammography MLO and CC views were assessed for the presence or absence of enhancing lesions. When an enhancing lesion was noted, it was then classified into mass or non-mass enhancement. In case of an enhancing mass lesion, further characterization was done by assessing its margins (circumscribed, not circumscribed irregular or not circumscribed spiculated), degree of enhancement (mild, moderate, and severe) and pattern of internal enhancement (homogenous, heterogeneous, septations, or ring enhancement). When a non-massenhancing lesion was noted, further characterization was done by assessing the distribution (focal, linear, segmental, regional, multiregional, or diffuse), pattern of internal enhancement (homogenous, heterogeneous, clustered, and clumped), and degree of enhancement (mild, moderate, and severe). We then determined the BIRADS category of each lesion in reference to MRI BIRADS atlas 2013 morphology descriptors [9] as there is no standardized BIRADS Lexicon to CEM.

On both modalities, each woman was given a BIRADS score for each breast. Yet just the higher BIRADS score (on both sides) was used to represent each woman on FFDM and CEM. The score was then compared with the pathology results which was used as the gold standard for 194 out of the 283 cases, and for the remaining 89 patients, ultrasound and routine follow-up were used for normal and typically benign findings (anechoic cysts, complicated cyst, adenosis, intramammary lymph node, and fibroadenoma). Cytological or histopathological analysis of the suspected breast lesions were obtained either by fine needle aspiration cytology, core biopsy, or surgery.

\section{Statistical analysis}

The statistical package SPSS (Statistical Package for the Social Sciences) version 25 was used for data coding and entering. Quantitative data were summarized using range, mean, and standard deviation and categorical data using frequency (count) and relative frequency (percentage). Standard diagnostic indices including sensitivity, specificity, positive predictive value (PPV), negative predictive value (NPV), and accuracy were calculated. Comparisons of quantitative variables between groups were done using unpaired $t$ test. Chi square $\left(x^{2}\right)$ test was performed for comparing categorical data, and when the expected frequency was less than 5, exact test was used instead.
Finally, statistically significant was considered when $P$ value was less than 0.05 .

\section{Results}

Two hundred eighty three women with increased risk of developing breast cancer were included in this prospective study. The mean age of this study population was 48 years (range 19-75 years).

Of the total study population, 156/283 (55.1\%) women came for diagnostic purpose, and 127/283 (49.9\%) women came for screening purpose. Out of the diagnostic cases, 109/156 (69.9\%) came presenting with palpable masses.

Among the studied cases regardless to a specific risk factor, $15 / 283(5.3 \%)$ cases were diagnosed as normal, $13 / 283(4.6 \%)$ as inflammatory lesions, $72 / 283(25.4 \%)$ as benign lesions, $6 / 283(2.1 \%)$ as benign precancerous lesions, and 177/283 (62.5\%) as malignant. Final diagnosis of the studied cases was shown in Table 2.

Table 2 Final diagnosis of the studied cases (proven by histopathology or ultrasound findings with routine follow-up for normal and typically benign findings)

\begin{tabular}{lll}
\hline Final diagnosis & Count & $\%$ \\
\hline Normal & $15 / 283$ & $5.3 \%$ \\
Adenosis & $14 / 283$ & $5 \%$ \\
Focal adenosis/hamartoma & $2 / 283$ & $0.7 \%$ \\
Intramammary lymph node & $1 / 283$ & $0.35 \%$ \\
Duct ectasia & $1 / 283$ & $0.35 \%$ \\
Fibrocystic changes & $12 / 283$ & $4.2 \%$ \\
Postoperative changes (scar, seroma, fat necrosis) & $12 / 283$ & $4.2 \%$ \\
Complicated cyst & $2 / 283$ & $0.7 \%$ \\
Fibroadenoma & $25 / 283$ & $8.8 \%$ \\
Lactating adenoma & $2 / 283$ & $0.7 \%$ \\
Papilloma & $1 / 283$ & $0.35 \%$ \\
Mastitis (lactational, periductal, granulomatous) & $10 / 283$ & $3.5 \%$ \\
Infected cyst & $1 / 283$ & $0.35 \%$ \\
Abscess & $2 / 283$ & $0.7 \%$ \\
Sclerosing adenosis & $1 / 283$ & $0.35 \%$ \\
Papillomatosis & $1 / 283$ & $0.35 \%$ \\
Atypical papillary proliferation & $1 / 283$ & $0.35 \%$ \\
Atypical ductal hyperplasia & $1 / 283$ & $0.35 \%$ \\
Lobular hyperplasia & $1 / 283$ & $0.35 \%$ \\
Phylloides & $1 / 283$ & $0.35 \%$ \\
DCIS & $9 / 283$ & $3.2 \%$ \\
IDC & $152 / 283$ & $53.7 \%$ \\
ILC/IDC & $1 / 283$ & $0.35 \%$ \\
ILC & $10 / 283$ & $3.5 \%$ \\
ITC & $2 / 283$ & $0.7 \%$ \\
Intraductal papillary neoplasm & $1 / 283$ & $0.35 \%$ \\
Pagets with DCIS & $2 / 283$ & $0.7 \%$ \\
\hline
\end{tabular}


Table 3 The final BIRADS of conventional versus contrast-enhanced mammography in correlation with final pathology

\begin{tabular}{|c|c|c|c|c|c|c|}
\hline & & \multicolumn{4}{|c|}{ Pathology } & \multirow[t]{3}{*}{$P$ value } \\
\hline & & \multicolumn{2}{|c|}{ Non-malignant } & \multicolumn{2}{|c|}{ Malignant } & \\
\hline & & Count & $\%$ & Count & $\%$ & \\
\hline \multirow{5}{*}{$\begin{array}{l}\text { FINAL MAMMO } \\
\text { BIRADS }\end{array}$} & Normal & 9 & $8.5 \%$ & 9 & $5.1 \%$ & \multirow[t]{5}{*}{$>0.001$} \\
\hline & Benign & 4 & $3.8 \%$ & 2 & $1.1 \%$ & \\
\hline & $\begin{array}{l}\text { Probably } \\
\text { benign }\end{array}$ & 49 & $46.2 \%$ & 23 & $13.0 \%$ & \\
\hline & Suspicious & 38 & $35.8 \%$ & 68 & $38.4 \%$ & \\
\hline & Malignant & 6 & $5.7 \%$ & 75 & $42.4 \%$ & \\
\hline \multirow{5}{*}{$\begin{array}{l}\text { FINAL CONTRAST } \\
\text { BIRADS }\end{array}$} & Norma & 26 & $24.5 \%$ & 3 & $1.7 \%$ & \multirow[t]{5}{*}{$>0.001$} \\
\hline & Benign & 334 & $32.1 \%$ & 4 & $2.3 \%$ & \\
\hline & $\begin{array}{l}\text { Probably } \\
\text { benign }\end{array}$ & 15 & $14.2 \%$ & 7 & $4.0 \%$ & \\
\hline & Suspicious & 12 & $11.3 \%$ & 21 & $11.9 \%$ & \\
\hline & Malignant & 19 & $17.9 \%$ & 142 & $80.2 \%$ & \\
\hline
\end{tabular}

One hundred seventy seven out of the $283(62.5 \%)$ cases were malignant, and 106/283 (37.5\%) cases were normal or benign lesions. Out of the malignant cases, 21/177 had bilateral malignant process, and 28/177 had multiple ipsilateral malignant lesions (multifocal or multicentric).

Mean age of patients with malignant mass lesions was 48.5 years $( \pm 11.6)$, and the mean age of those with benign lesions was 47.8 years $( \pm 11.1)$. No statistical significant difference was identified between both groups ( $P$ value 0.622 ). Eleven out of the total population were aged less than 30 years. All of them had suspicious lesions (BIRADS 4 or 5) on their initial ultrasound scan.

Twenty four out of the 283 (8.5\%) cases were ACR A and B (non-dense), while 259/283 (91.5\%) cases were ACR C and D (dense).

Eighty four out of the 283 (29.7\%) women had positive family history (one or more first-degree relative); 199/ $283(70.3 \%)$ had no family history.

Two hundred thirty two out of the 283 (82.0\%) women had children, and 51/283(18.0\%) had no children.

Two hundred thirty nine out of the 283 (84.5\%) women had history of breast feeding, and 44/283 (15.5\%) gave history of artificial feeding.

Seventy three out of the 283 (25.8\%) women had history of hormonal contraception use, and 210/283 (74.2\%) had not.

One hundred and seven out of the 283 (37.8\%) women were menopausal, 176/283 (62.2\%) women were not.

Sixteen out of the 283 (5.7\%) women having previous breast operation, and 267/283 (94.3) did not have previous operation.
No significant correlation was calculated between previous factors and the presence of malignant lesions.

All patients had a BIRADS category according to the sonomammography:

- Eighteen of the 283 (6.4\%) cases were assigned BIRADS 1 (normal).

- Six of the 283 (2.1\%) cases were assigned BIRADS 2 (benign).

- Seventy two of the 283 (25.4\%) cases were assigned BIRADS 3 (probably benign).

- One hundred six of the 283 (37.5\%) cases were assigned BIRADS 4 (suspicious lesions).

- Eighty one of the 283(28.6\%) cases were assigned BIRADS 5 (malignant).

Total study population (283 women) underwent contrastenhanced mammography and the BIRADS category were according to CEM:

Table 4 Accuracy measures of FFDM and CEM

\begin{tabular}{lll}
\hline & Count & $\%$ \\
\hline FFDM accuracy final & & \\
TP & 144 & $50.9 \%$ \\
FP & 43 & $15.2 \%$ \\
TN & 62 & $21.9 \%$ \\
FN & 34 & $12.0 \%$ \\
CESM accuracy final & & \\
TP & 165 & $85.3 \%$ \\
FP & 30 & $10.6 \%$ \\
TN & 75 & $26.5 \%$ \\
FN & 13 & $4.5 \%$ \\
\hline
\end{tabular}


Table 5 Diagnostic indices of FFDM and CEM

\begin{tabular}{lll}
\hline & Value & 95\% Cl \\
\hline FFDM & & \\
Sensitivity & $80.9 \%$ & $74.34 \%$ to $86.39 \%$ \\
Specificity & $59.05 \%$ & $49.02 \%$ to $68.55 \%$ \\
Positive predictive value & $77.01 \%$ & $72.47 \%$ to $80.99 \%$ \\
Negative predictive value & $64.58 \%$ & $56.44 \%$ to $71.96 \%$ \\
Accuracy & $72.79 \%$ & $67.21 \%$ to $77.89 \%$ \\
CESM & & \\
Sensitivity & $92.7 \%$ & $87.83 \%$ to $96.05 \%$ \\
Specificity & $71.43 \%$ & $61.79 \%$ to $79.82 \%$ \\
Positive predictive value & $84.62 \%$ & $80.21 \%$ to $88.18 \%$ \\
Negative predictive value & $85.23 \%$ & $77.12 \%$ to $90.80 \%$ \\
Accuracy & $84.81 \%$ & $80.08 \%$ to $88.78 \%$ \\
\hline
\end{tabular}

- Twenty nine of the 283 (10.2\%) cases were assigned BIRADS 1 (normal).

- Thirty eight of the 283 (13.4\%) cases were assigned BIRADS 2 (benign).

- Twenty two of the 283 (7.8\%) cases were assigned BIRADS 3 (probably benign).

- Thirty three of the 283 (11.7\%) cases were assigned BIRADS 4 (suspicious lesions).

- One hundred sixty one of the 283(56.9\%) cases were assigned BIRADS 5 (malignant).

The final BIRADS of conventional versus contrastenhanced mammography in correlation with final pathology was shown in Table 3.

The accuracy measures and diagnostic indices of both FFDM and CEM are shown in Tables 4 and 5.

\section{Discussion}

Mammography is the primary imaging modality in screening and early detection of breast cancer, yet it still

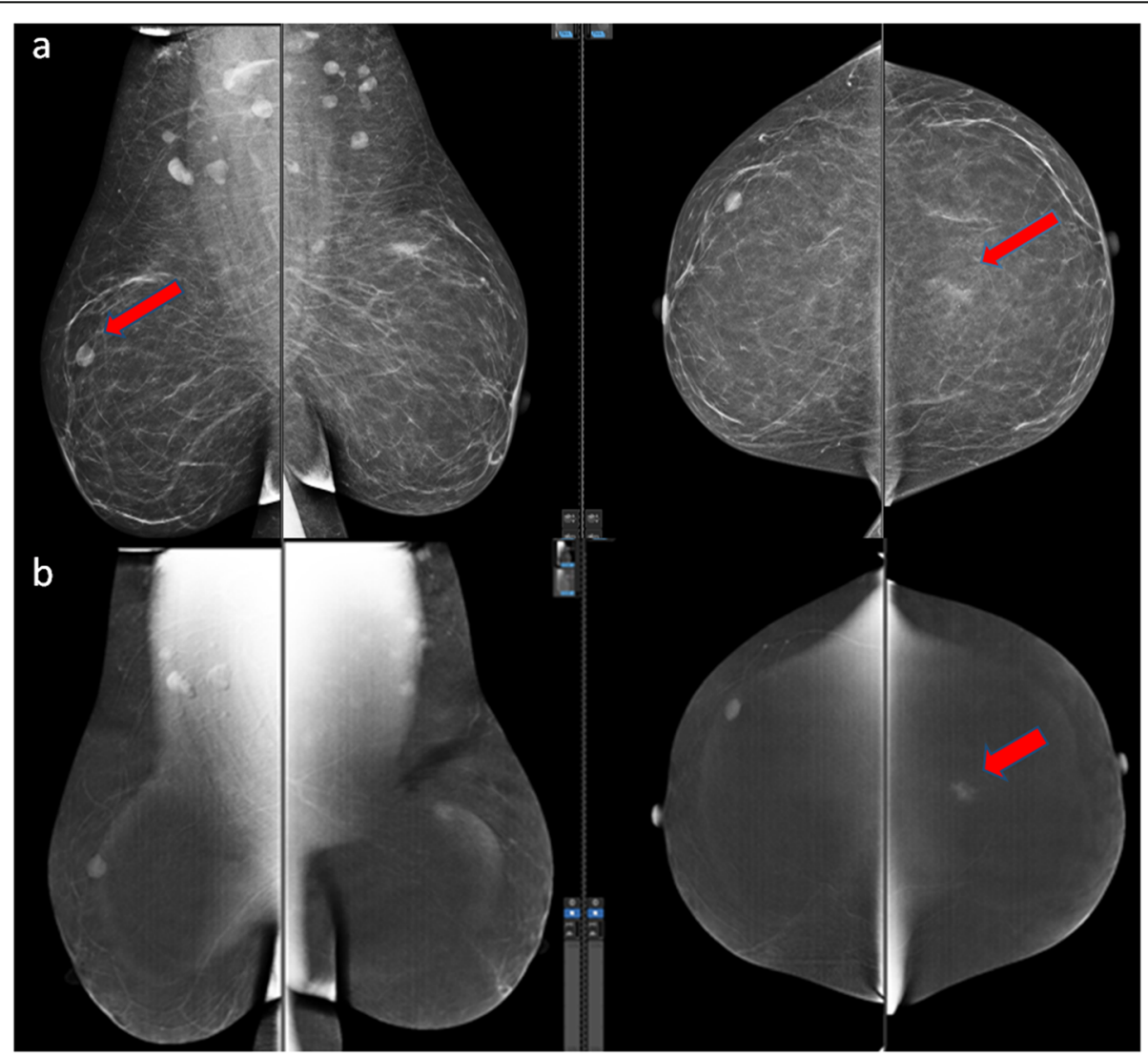

Fig. 1 A 49-year-old female coming for screening with history of hormonal contraception intake. a FFDM showed scattered fibro-glandular densities (ACR B), right oval-shaped well-circumscribed lesion (BIRADS 3) and left focal asymmetry with underlying subtle irregular-shaped lesion (BIRADS 4). b CESM showed homogeneous enhancement of the right breast lesion (BIRADS 2) and heterogeneous enhancement with spiculated margins of the left breast lesion (BIRADS 5). Histopathology: left breast IDC; US with follow-up: right breast small adenoma. Comment: CEM confirmed the malignant and benign nature of both lesions seen on mammography 
has its fallacies. Its limitations, particularly in women with dense breasts and/or at risk for breast cancer, have led to the invention of improved and tailored techniques to patient risk [10].

Breast MRI is currently considered to be the most sensitive imaging modality in the detection of breast cancer with sensitivity approaching $95-100 \%$, as proved by large trials to screen high-risk women [11]. The American College of Radiology recommends annual screening breast MRI in addition to an annual mammogram in women at high risk of developing breast cancer and, more recently, those with a personal history of breast cancer who have dense breasts or those diagnosed before age 50 years among women with a $15-20 \%$ lifetime risk of breast cancer [5].

Contrast-enhanced MRI-combined morphofunctional approach enforces the basis of the vascular-based technique; CEM, which can possibly serve as its alternative $[10,12]$. CEM images reliably enhanced diagnostic performance when compared with digital mammography, ultrasound, and DBT, with overall performance comparable with CE-MRI [13].
Since women with BRCA mutations have increased risk of interval cancers, some of them stagger screening mammography and MRI at 6-month intervals instead of performing both examinations on the same day (stacked screening) [14]. Furthermore, in such patients with high risk for breast cancer, CEM can replace conventional FFDM allowing contrast-enhanced screening test to be performed every 6 months [10]. It was observed that in high-risk women screening, CEM is much better tolerated than CE-MRI due to its shorter examination time [15].

In the current study, we assessed the additive role of contrast-enhanced mammography in patients with increased risk of developing breast cancer and its capability in overcoming mammographic deficiencies.

In this prospective study, we included 283 consecutively registered women (with increased risk of developing breast cancer) who underwent screening (127/283 (49.1\%)) or diagnostic (156/283 (55.1\%)) FFDM and CEM, we have found that $177 / 283(62.5 \%)$ cases had malignant lesions (Fig. 1), and 106/283 (37.5\%) cases had benign lesions (Fig. 2).

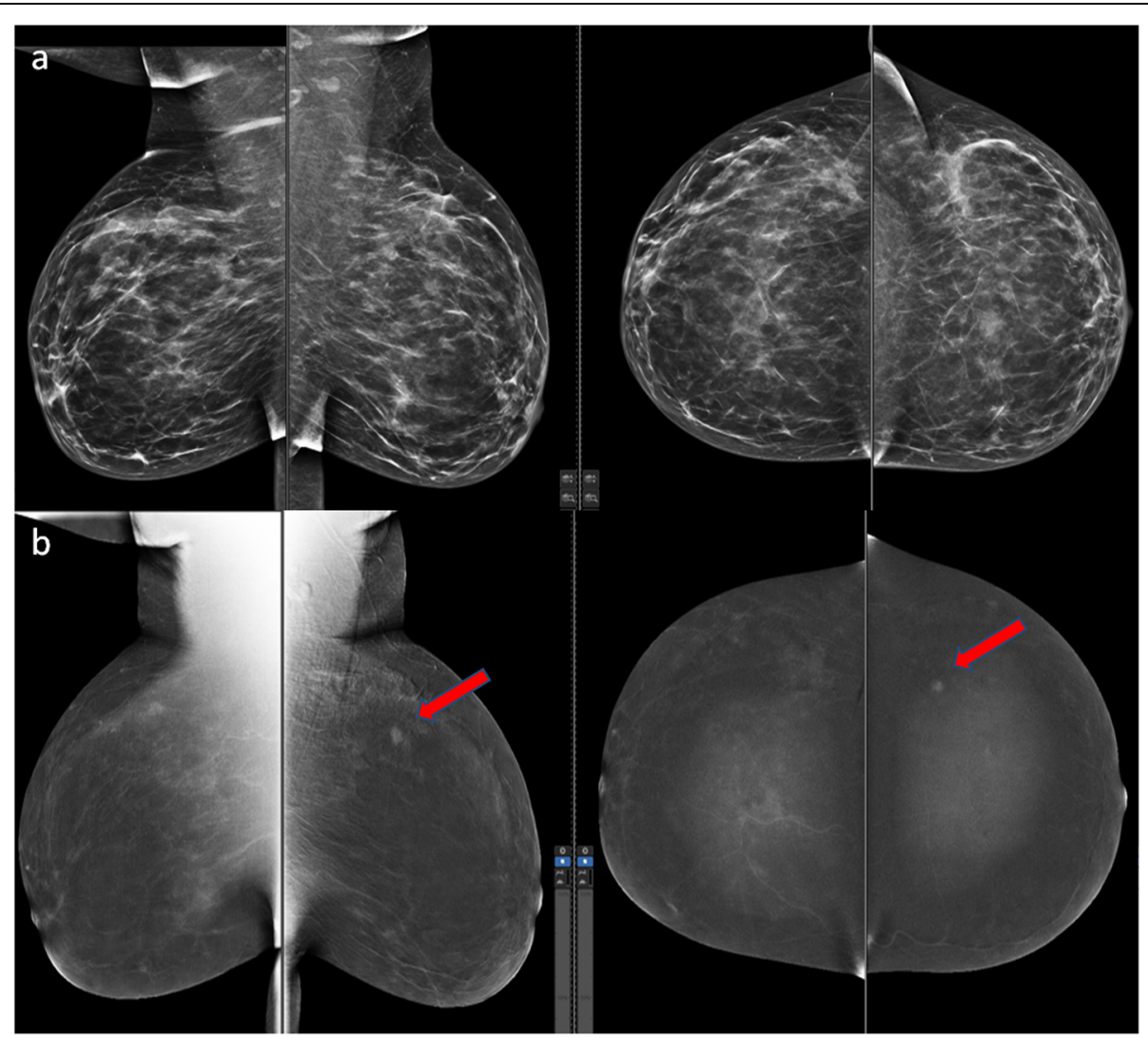

Fig. 2 A 42-year-old female coming for screening with family history of breast cancer (mother and aunt). a FFDM showed heterogeneously dense fibro-glandular parenchyma (ACR C), no right or left breast lesions (BIRADS 1). b CESM showed no right breast lesions (BIRADS 1) and left UOQ small enhancing circumscribed lesion (BIRADS 3). Histopathology: left breast fibroadenoma. Comment: CESM showed an underlying lesion not seen in mammography 
In our study population, none of the factors we studied correlated with breast cancer development. This might be attributed to the limited number of cases included in our study.

In contrast to Ozsoy et al., where they concluded that among the risk factors for breast cancer, the presence of breast cancer in family was found to be the most important risk factor. They also stated that there was no relationship identified in terms of the presence and duration of breastfeeding, the use of OCP, and the use of HRT and breast cancer risk [16].

The mean age in our study in patients with malignant lesions was $48.5 \pm 11.5$, and it is much lower than that reported in other studies $[16,17]$. This matches with the reported age incidence in Egypt which is 10 years younger than Europe and USA [18].

In the current study, CEM increased the accuracy of BIRADS assessment of malignant cases (Figs. 3 and 4) and also helped in the detection of patients with multifocal and multicentric lesions.
In view of our results, contrast-enhanced mammography technique has the potential to improve characterization of breast lesions (Fig. 5). It contributed to marked reduction in the number of false-positive and false-negative cases. The sensitivity, specificity, and positive predictive value of CEM were 92.7, 71.4, and 84.6\%, respectively, and of mammography were $80.9,59.0$, and $77.0 \%$, respectively.

These results are supported by Dromain et al.'s study, which was performed on 110 patients with 148 breast lesions. They confirmed the superior diagnostic accuracy of CEM in addition to sonomammography with sensitivity of $93 \%$ [19].

Luczyńska et al. also concluded that contrast-enhanced mammogram has improved sensitivity and specificity in breast cancer detection through a study performed on 152 consecutive patients with 173 breast lesions diagnosed on conventional mammogram or CEM [20]. ElSaid et al. also agreed with these results [21].

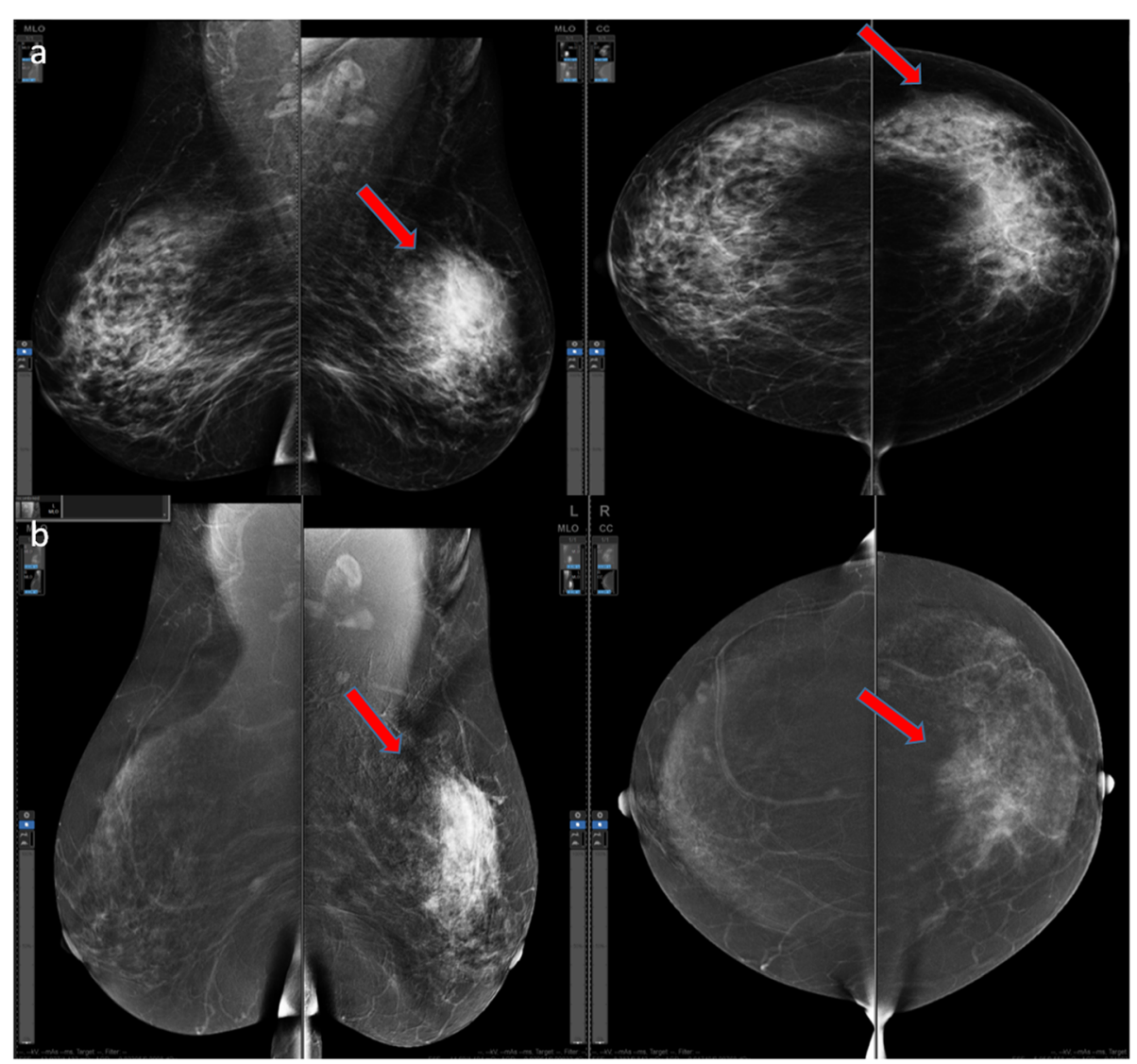

Fig. 3 A 48-year-old female coming from the left breast lump with heterogeneous dense breasts. a FFDM showed heterogeneously dense fibroglandular parenchyma ( $A C R C$ ), no right breast lesions (BIRADS 1) and left UOQ focal asymmetry with left retracted nipple (BIRADS 4). b CESM showed no right-enhancing lesions (BIRADS 1) and left heterogeneous segmental non-mass enhancement seen radiating from the nipple (with the line of cleavage) backwards with left retracted nipple (BIRADS 5). Histopathology: left breast IDC2. Comment: CESM proved the malignant nature of breast lesion and showed an underlying lesion not seen in mammography 

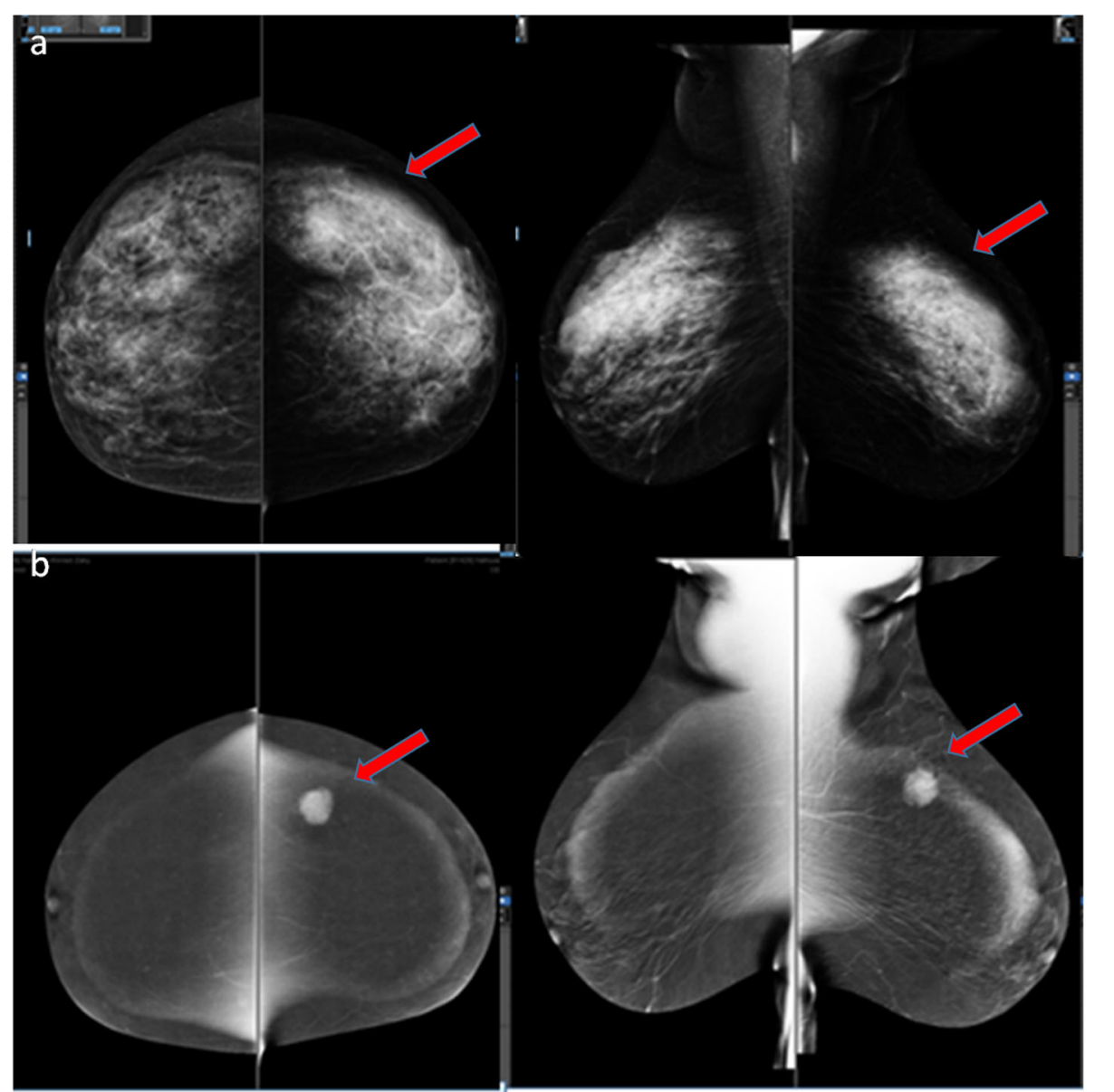

Fig. 4 A 68-year-old female coming complaining from the left breast lump with heterogeneously dense breasts. a FFDM showed heterogeneously dense fibro-glandular parenchyma (ACR C), normal right breast (BIRADS 1) and left global asymmetry (BIRADS 4). b CESM showed no right breast-enhancing lesions (BIRADS 1) and left UOQ intensely enhancing spiculated lesion (BIRADS 5). Histopathology: left breast IDC.

Comment: CESM showed underlying lesion not seen in mammography

Sung et al. recently stated that CEM has the potential to be an alternative screening technique to twodimensional full-field in women at increased risk of breast cancer with sensitivity of $87.5 \%$ (compared with $50.0 \%$ for FFDM) and specificity of $93.7 \%$ [10].

In our study, CEM was of special value in patients with dense breast tissue in both screening and nonscreening context giving a greater diagnostic confidence and less operator dependency than mammography alone (Figs. 3, 4 and 5). It also acted as a problem-solving technique, decreasing the number of unnecessary biopsies in cases with intermediate lesions (BIRADS 3 or 4 lesions on mammography) such as areas of asymmetry with no underlying ultrasound findings.

Our study had some limitations; the fundamental one was that the cases included in the study were not cases that participated in the general community screening, but were cases that presented to our hospital for opportunistic screening or diagnostic purpose mostly upon clinician's referral. There may be statistical differences between general community screening cases and cases that presented to the hospital.

The other limitation was the limited number of cases included in the study. Studies with larger series are needed to be able to clearly identify the risk factors related to the community in Egypt.

The last and major limitation was the selection of cases, which was only based on modifiable and nonmodifiable risk factors and not BRCA gene mutation.

Contrast-enhanced mammography is a promising technique that deserves all the attention previously and currently given to MRI and that is why further multicenter studies with large number of patients is needed to validate the positive CEM results. 


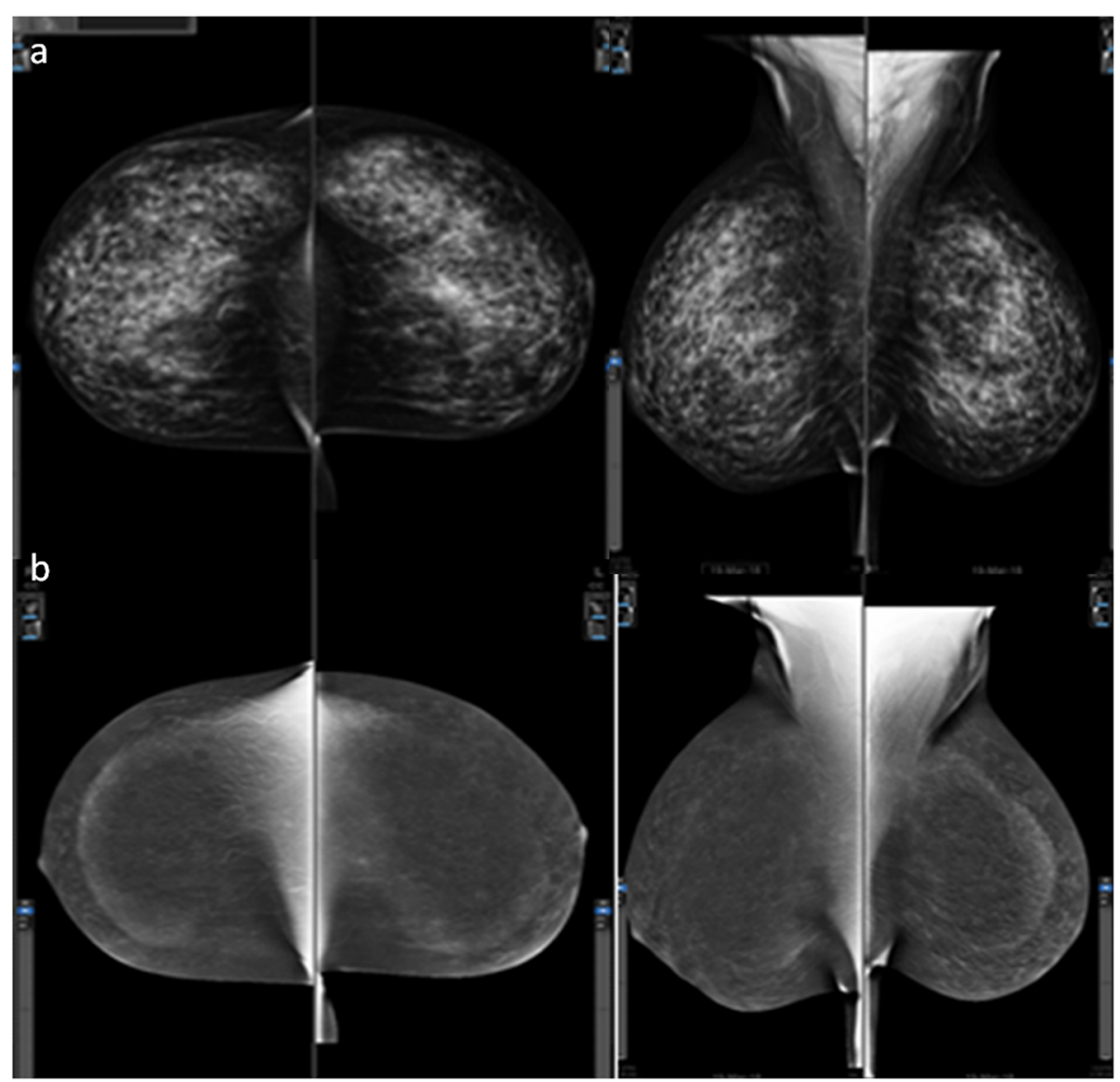

Fig. 5 A 43-year-old female coming for screening with dense breasts and positive family history (sister). a FFDM showed dense fibro-glandular parenchyma (ACR D), multiple, bilateral, scattered suspected well-defined nodules (BIRADS 3). b CESM showed bilateral faintly enhancing multiple foci (4 mm) (BIRADS 2). US and follow-up: bilateral adenosis. Comment: CESM proved the benign nature of breast lesions

\section{Conclusion}

Contrast-enhanced mammography is a valuable screening and diagnostic imaging modality in patients with increased risk of developing breast cancer with diagnostic indices higher than mammography resulting in a significantly higher cancer detection rate.

\section{Abbreviations}

CEM: Contrast-enhanced mammography; FFDM: Full-field digital mammography; BIRADS: Breast imaging reporting and data system; MRI: Magnetic resonance imaging; CE-MRI: Contrast-enhanced magnetic resonance imaging; CC: Cranio-caudal; MLO: Mediolateral oblique; ACR: American College of Radiology; DBT: Digital breast tomosynthesis; BRCA: Breast cancer gene; OCP: Oral contraceptive pills; HRT: Hormone replacement therapy

\section{Acknowledgements}

No acknowledgements.

\section{Authors' contributions}

YT wrote the manuscript. NH collected patient data. LH worked on image processing and collection of patient's images. RK participated in the design of the study and performed the statistical analysis. $\mathrm{NH}$ and $\mathrm{LH}$ conceived the study and participated in its design and coordination and helped to draft the manuscript. RK and SM were responsible for the revision of the draft from clinical point of view. SL collected the clinical and pathological data and statistics analysis. The authors have read and approved the manuscript.

\section{Funding}

No funding sources.

\section{Availability of data and materials}

The data sets used and analyzed during the current study are available from the corresponding author on reasonable request.

\section{Declarations}

Ethics approval and consent to participate

The study was approved by the ethical committee of "Faculty of Medicine, Cairo University" with ethical committee approval number and date not available. An informed written consent was taken from all subjects.

\section{Consent for publication}

All patients included in this research gave written informed consent to publish the data contained within this study.

\section{Competing interests}

No financial or non-financial competing interests 


\section{Author details}

'Department of Diagnostic and Interventional Radiology, Faculty of Medicine, Cairo University, Cairo, Egypt. ${ }^{2}$ Department of Diagnostic and Interventional Radiology, Baheya Foundation, Cairo, Egypt. ${ }^{3}$ Department of Diagnostic and Interventional Radiology, El Shaikh Zayed Hospital, Cairo, Egypt. ${ }^{4}$ Department of Clinical Oncology, Faculty of Medicine, Cairo University, Cairo, Egypt.

Received: 27 October 2020 Accepted: 22 February 2021

Published online: 03 March 2021

\section{References}

1. Gradishar WJ, Anderson BO, Balassanian R et al (2015) Breast cancer version 2.2015. J Natl Compr Canc Netw 13(4):448-475

2. Lodish H, Berk A, Zipursky SL et al (2000) DNA damage and repair and their role in carcinogenesis. In: Molecular cell biology, 4th edn, p 12.4

3. Freer PE (2015) Mammographic breast density: impact on breast cancer risk and implications for screening. RadioGraphics. 35:302-315

4. Lee Cl, Chen LE, Elmore JG (2017) Risk-based breast cancer screening: implications of breast density. Med Clin North Am 101(4):725-741

5. Monticciolo DL, Newell MS, Moy L et al (2018) Breast cancer screening in women at higher-than-average risk: recommendations from the ACR. J Am Coll Radiol 15:408-414

6. Cozzi A, Schiaffino S, Sardanelli F (2019) The emerging role of contrastenhanced mammography. Quant Imaging Med Surg 9(12):2012-2018

7. Jochelson MS, Dershaw DD, Sung JS et al (2013) Bilateral contrast-enhanced dual-energy digital mammography: feasibility and comparison with conventional digital mammography and MR imaging in women with known breast carcinoma. Radiology. 266:743-751

8. Kamal RM, Helal MH, Mansour SM et al (2016) Can we apply the MRI BIRADS lexicon morphology descriptors on contrast-enhanced spectral mammography? Br J Radiol 89(1064):20160157

9. Morris EA, Comstock CE, Lee CH et al (2013) ACR BI-RADS magnetic resonance imaging. In: ACR BI-RADS ${ }^{\circledR}$ atlas, breast imaging reporting and data system. American College of Radiology, Reston

10. Sung JS, Lebron L, Keating D et al (2019) Performance of dual-energy contrast-enhanced digital mammography for screening women at increased risk of breast cancer. Radiology. 293:81-88

11. Mann RM, Kuhl CK, Moy L (2019) Contrast-enhanced MRI for breast cancer screening. J Magn Reson Imaging 50:377-390

12. Ghaderi KF, Phillips J, Perry H et al (2019) Contrast-enhanced mammography: current applications and future directions. Radiographics. 39(7):1907-1920

13. Dromain C, Vietti-Violi N, Meuwly JY (2019) Angiomammography: a review of current evidences. Diagn Interv Imaging 100(10):593-605

14. Lowry KP, Lee JM, Kong CY et al (2012) Annual screening strategies in BRCA1 and BRCA2 gene mutation carriers: a comparative effectiveness analysis. Cancer. 118(8):2021-2030

15. Phillips J, Miller MM, Mehta TS et al (2017) Contrast-enhanced spectral mammography (CESM) versus MRI in the high-risk screening setting: patient preferences and attitudes. Clin Imaging 42:193-197

16. Ozsoy A, Barca N, Dolek BA et al (2017) The relationship between breast cancer and risk factors: a single-center study. Eur J Breast Health 13(3):145-149

17. Singletary SE (2003) Rating the risk factors for breast cancer. Ann Surg 237(4):474-482

18. Ibrahim AS, Khaled HM, Mikhail NNH et al (2014) Cancer incidence in Egypt: results of the National Population-Based Cancer Registry Program. J Cancer Epidemiol 2014:437971, https://doi.org/10.1155/2014/437971 Epub 2014 Sep 21. PMID: 25328522; PMCID: PMC4189936, https://pubmed.ncbi.nlm.nih. gov/25328522/.

19. Dromain C, Thibault F, Diekmann F et al (2012) A dual-energy contrastenhanced digital mammography: initial clinical results of a multireader, multicase study. Breast Cancer Res 14(3):R94

20. Luczyńska E, Heinze-Paluchowska S, Dyczek S et al (2014) Contrast-enhanced spectral mammography: comparison with conventional mammography and histopathology in 152 women. Korean J Radiol 15(6):689-696

21. ElSaid N, Farouk S, Shetat O et al (2015) Contrast enhanced digital mammography: is it useful in detecting lesions in edematous breast? Egypt J Radiol Nucl Med 46:811-819

\section{Publisher's Note}

Springer Nature remains neutral with regard to jurisdictional claims in published maps and institutional affiliations.

\section{Submit your manuscript to a SpringerOpen ${ }^{\circ}$ journal and benefit from:}

- Convenient online submission

- Rigorous peer review

- Open access: articles freely available online

- High visibility within the field

- Retaining the copyright to your article

Submit your next manuscript at $\boldsymbol{\nabla}$ springeropen.com 